\title{
The clinicopathological features of sinonasal angiomatous polyps
}

\section{Yuan-Yun Tam ${ }^{1, *}$ \\ Chia-Chen $\mathrm{Wu}^{2, *}$ \\ Ta-Jen Lee ${ }^{2}$ \\ Yang-Yu Lin ${ }^{3}$ \\ Tai-Di Chen ${ }^{4}$ \\ Chi-Che Huang ${ }^{2}$}

'Department of Otorhinolaryngology Head and Neck Surgery, Lotung

Poh-Ai Hospital, Yilan County,

${ }^{2}$ Department of Otorhinolaryngology -

Head and Neck Surgery, ${ }^{3}$ Department

of Medical Imaging and Intervention,

${ }^{4}$ Department of Pathology, Linkou

Chang Gung Memorial Hospital,

Chang Gung University, Taoyuan

County, Taiwan

*These authors contributed equally to this work

This article was published in the following Dove Press journal:

International Journal of General Medicine

17 June 2016

Number of times this article has been viewed

Background: Sinonasal angiomatous polyp (SAP) is a rare subtype of sinonasal polyp that might be misdiagnosed as a malignant lesion due to its clinical symptoms.

Methods: We retrospectively enrolled the patients who were diagnosed with SAP in our hospital during 2008-2015. We analyzed the clinical symptoms, radiological findings, and pathological features of all patients diagnosed with SAP.

Results: Unilateral nasal obstruction, rhinorrhea, and epistaxis were the common symptoms. SAPs all originated from maxillary sinus and extended to nasal cavity with or without involving the nasopharynx. Expansile mass with surrounding bony destruction is typical on computed tomography imaging but specific for SAPs. The magnetic resonance revealed high signal intensity on T1-weighted images and hypointense rim on T2-weighted images.

Conclusion: Computed tomography and magnetic resonance together might give rise to more accurate diagnosis of SAP. Incisional biopsy does help if the clinician suspects a malignant lesion. To treat SAP, complete removal is the optimal choice.

Keywords: sinonasal angiomatous polyp, angiomatous polyp, antrochoanal polyp, angiomatous nasal polyp, infarcted nasal polyp

\section{Introduction}

Sinonasal angiomatous polyp (SAP), a subtype of sinonasal polyp, is a benign and nonneoplastic lesion rarely reported in the literature. ${ }^{1}$ It is also known as angiectatic polyp and is characterized by extensive vascular proliferation and angiectasis with regions that are susceptible to vascular compromise, resulting in venous stasis, thrombosis, and infarction. ${ }^{2}$ Its clinical and radiological features may simulate those of neoplastic lesions such as juvenile angiofibroma, inverted papilloma, hemangioma, and even malignant sinonasal tumor. ${ }^{3}$ However, SAP can be treated with conservative surgical excision and recurrence is rare. Several studies discussed the findings of SAPs on computed tomography (CT) and magnetic resonance (MR) imaging, ${ }^{4,5}$ but there are scant reports describing the clinical features of the disease. To the best of our knowledge, there are only a few case reports describing SAP., ${ }^{3,67}$ In this article, we present a clinicopathological and radiological study of 13 patients with pathologically proven SAP.

Correspondence: Chi-Che Huang Department of Otorhinolaryngology Head and Neck Surgery, Linkou Chang Gung Memorial Hospital, Chang Gung University, Number 5, Fu Hsing Street, Kwei-Shan, Taoyuan County 333, Taiwan Tel +88633281200 ext8466

Fax $+8863327 \quad 1244$

Email hcc3110@adm.cgmh.org.tw

\section{Materials and methods}

The institutional review board of the Chang Gung Memorial Hospital approved this retrospective study and did not require patient consent be obtained, as this was a retrospective study, and all data was de-identified. We enrolled 13 patients who were 
pathologically diagnosed with SAP from 2008 to 2015 and reviewed their clinical presentation, including sex, age, symptoms and signs, sinuscopic findings, medical history, and radiologic images, including $\mathrm{CT}$ and MR. All patients underwent a preoperative biopsy of nasal polypoid tumor or intraoperative biopsy for frozen section, due to the high index of suspicion for neoplasia, before surgical intervention. Functional endoscopic sinus surgery to remove the angiomatous polyps and to restore optimal sinus function was performed in all cases.

\section{Results}

\section{Clinical findings}

The demographic data of all 13 patients are presented in Table 1. There were six females and seven males. Their ages ranged from 12 years to 72 years, with a mean age of $46.4 \pm 19.7$ years. All SAPs were unilateral, eight on the left and five on the right side, with none affecting the contralateral sinonasal cavity. The patients presented with nasal obstruction $(11 / 13,84.6 \%)$, rhinorrhea $(12 / 13,92.3 \%)$, or epistaxis $(6 / 13,46.1 \%)$, all unilateral. Three patients had hyposmia, and two patients had visual disturbance. The duration of symptoms ranged from 3 months to 5 years. Six patients had type 2 diabetes mellitus, for which they were taking oral hypoglycemic agents. All patients denied any history of trauma or previous surgery. Four patients were taking aspirin, one because of a history of chronic atrial fibrillation and the other three for a history of cerebrovascular accident. One patient with diabetes mellitus also had end-stage renal disease. Seven of the patients had no other medical history. Only one patient was a smoker. All patients underwent sinuscopy on their first visit and before surgery. This revealed a huge polypoid mass obliterating the osteomeatal complex alternating with a reddish, bluish, or black necrotic area with a tendency to bleed on touching in eleven cases and a huge antrochoanal polyp extending to the nasopharynx in two cases (Figure 1). Twelve patients had a diagnostic incisional biopsy of the sinonasal polyps performed on suspicion of sinonasal malignancy. The incisional biopsy diagnoses were blood clot (3/13, 23.1\%), inflammatory polyp $(10 / 13,76.9 \%)$, and fibrous exudate $(5 / 13,38.5 \%)$. After surgery, most of the patients were free of preoperative symptoms including those with visual disturbance, however two patients still complained of hyposmia.

\section{Imaging studies}

All patients received sinus CT studies prior to surgery, and six patients $(46.2 \%)$ had additional MR studies on suspicion of sinonasal malignancy. On CT images, all SAPs had the following features: origination from a unilateral maxillary sinus, involvement of the osteomeatal complex, and obliteration of the nasal cavity. Other involved anatomical sites included the inferior orbital floor $(8 / 13,61.5 \%)$, ethmoid sinus $(6 / 13$, $46.2 \%)$, sphenoid sinus $(3 / 13,23.1 \%)$, choana $(2 / 13,15.4 \%)$, nasopharynx $(1 / 13,7.7 \%)$, and infratemporal fossa $(1 / 13$,

Table I Demographic and clinical data

\begin{tabular}{|c|c|c|c|c|c|c|c|c|c|c|c|c|c|}
\hline \multirow{2}{*}{$\begin{array}{l}\text { Patient } \\
\text { number }\end{array}$} & \multirow[t]{2}{*}{ Sex } & \multirow{2}{*}{$\begin{array}{l}\text { Age, } \\
\text { years }\end{array}$} & \multirow{2}{*}{$\begin{array}{l}\text { Duration } \\
\text { of } \\
\text { symptoms }\end{array}$} & \multicolumn{5}{|c|}{ Clinical symptoms } & \multirow[t]{2}{*}{ History } & \multirow{2}{*}{$\begin{array}{l}\text { Anticoagulant } \\
\text { use }\end{array}$} & \multirow[t]{2}{*}{ CT } & \multirow[t]{2}{*}{ MR } & \multirow[t]{2}{*}{ Laterality } \\
\hline & & & & $\begin{array}{l}\text { Nasal } \\
\text { obstruction }\end{array}$ & Hyposmia & Rhinorrhea & Epistaxis & $\begin{array}{l}\text { Visual } \\
\text { disturbance }\end{array}$ & & & & & \\
\hline I & $\mathrm{F}$ & 52 & $\mathrm{l} \mathrm{yr}$ & $\mathrm{Y}$ & $Y$ & $Y$ & $N$ & $Y$ & None & None & $Y$ & $Y$ & Left \\
\hline 2 & $M$ & 47 & $\mathrm{l} y \mathrm{r}$ & $\mathrm{Y}$ & $N$ & $Y$ & $Y$ & $\mathrm{~N}$ & $\begin{array}{l}\text { Previous } \\
\text { CVA/ } \\
\text { DM }\end{array}$ & Aspirin & $Y$ & $Y$ & Left \\
\hline 3 & $\mathrm{~F}$ & 62 & $6 \mathrm{mo}$ & $N$ & $\mathrm{~N}$ & $Y$ & $Y$ & $\mathrm{~N}$ & $\begin{array}{l}\text { DM/atrial } \\
\text { fibrillation }\end{array}$ & Aspirin & $Y$ & $Y$ & Left \\
\hline 4 & $M$ & 72 & $\mathrm{l} y \mathrm{r}$ & $N$ & $N$ & $\mathrm{~N}$ & $Y$ & $Y$ & DM & None & $Y$ & $Y$ & Right \\
\hline 5 & $M$ & 30 & $6 \mathrm{mo}$ & $Y$ & $\mathrm{~N}$ & $Y$ & $N$ & $\mathrm{~N}$ & None & None & $\mathrm{Y}$ & $\mathrm{N}$ & Left \\
\hline 6 & $M$ & 66 & $6 \mathrm{mo}$ & $Y$ & $N$ & $Y$ & $Y$ & $\mathrm{~N}$ & $\begin{array}{l}\text { Previous } \\
\text { CVA/ } \\
\text { DM }\end{array}$ & Aspirin & $Y$ & $Y$ & Left \\
\hline 7 & $\mathrm{~F}$ & 64 & $\mathrm{l} y \mathrm{r}$ & $Y$ & $Y$ & $Y$ & $\mathrm{~N}$ & $\mathrm{~N}$ & $\begin{array}{l}\text { Previous } \\
\text { CVA/ } \\
\text { DM }\end{array}$ & Aspirin & $Y$ & $\mathrm{~N}$ & Right \\
\hline 8 & $\mathrm{~F}$ & 51 & $3 y r$ & $Y$ & $N$ & $Y$ & $N$ & $\mathrm{~N}$ & None & None & $Y$ & $Y$ & Left \\
\hline 9 & $\mathrm{~F}$ & 12 & $3 \mathrm{yr}$ & $Y$ & $N$ & $Y$ & $N$ & $\mathrm{~N}$ & None & None & $Y$ & $\mathrm{~N}$ & Left \\
\hline 10 & $M$ & 17 & $3 \mathrm{yr}$ & $Y$ & $\mathrm{~N}$ & $Y$ & $N$ & $\mathrm{~N}$ & None & None & $y$ & $\mathrm{~N}$ & Right \\
\hline II & $M$ & 23 & $6 \mathrm{mo}$ & $Y$ & $Y$ & $Y$ & $Y$ & $N$ & None & None & $Y$ & $N$ & Right \\
\hline 12 & $\mathrm{~F}$ & 49 & $3 \mathrm{mo}$ & $Y$ & $N$ & $Y$ & $N$ & $\mathrm{~N}$ & None & None & $Y$ & $N$ & Left \\
\hline 13 & $\mathrm{~F}$ & 58 & $5 \mathrm{yr}$ & $Y$ & $\mathrm{~N}$ & $Y$ & $Y$ & $\mathrm{~N}$ & None & None & $Y$ & $N$ & Left \\
\hline
\end{tabular}

Abbreviations: CT, computed tomography; MR, magnetic resonance; CVA, cerebrovascular accident; DM, diabetes mellitus; $F$, female; M, male; yr, years; mo, months; $Y$, yes; $N$, no. 
$7.7 \%)$. The densities of the SAPs were heterogeneously high (mean 59.4 $\pm 6.7 \mathrm{HU}$, range 50.8-71.0 HU), and most masses $(12 / 13,92.3 \%)$ were expansile with bony remodeling and even erosion $(7 / 13,53.4 \%)$ to the adjacent bony structures (Figure 2). All the SAPs showed sinus expansion and bone remodeling. On MR images, all the SAPs $(n=6)$ were mildly hyperintense on T1-weighted images (T1WI), heterogeneously hyperintense on T2-weighted images (T2WI), and avidly enhanced on contrast-enhanced T1WI. Moreover, interspersed parts of SAPs, which showed hypointensity on T1WI, a signal void on T2WI, and no contrast enhancement, were noted in five patients. Peripheral hypointense rims $(n=6)$
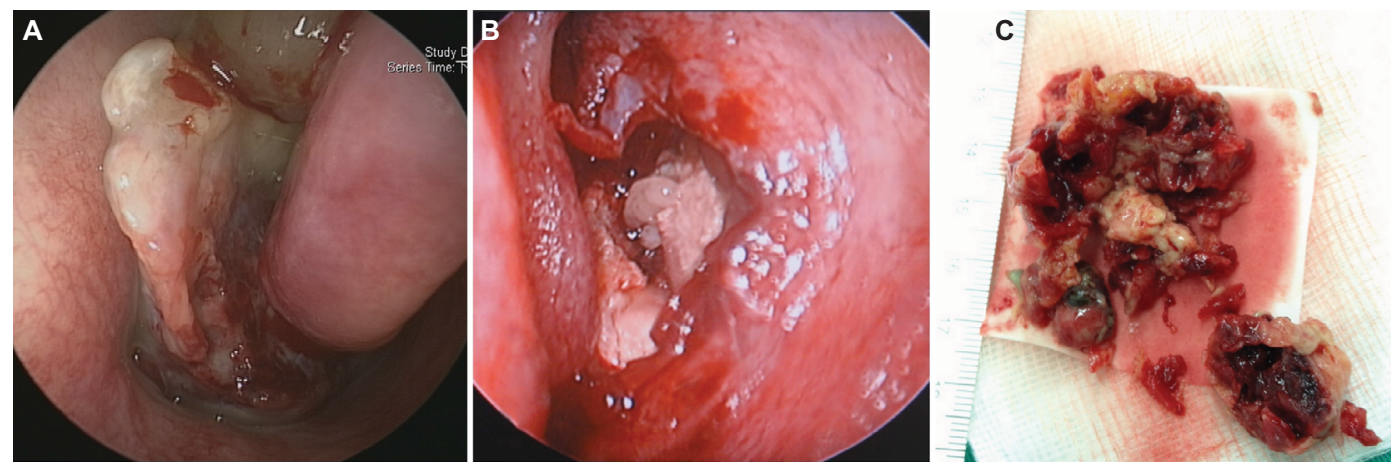

Figure I Clinical photography of sinonasal angiomatous polyp.

Notes: Sinuscopy revealed a huge antrochoanal polyp which bled easily when touched (A) with some reddish, bluish, necrotic gray area (B). A photo of SAP (C) shows gelatinous masses with glistening surfaces alternating gray and dark red color.

Abbreviation: SAP, sinonasal angiomatous polyp.

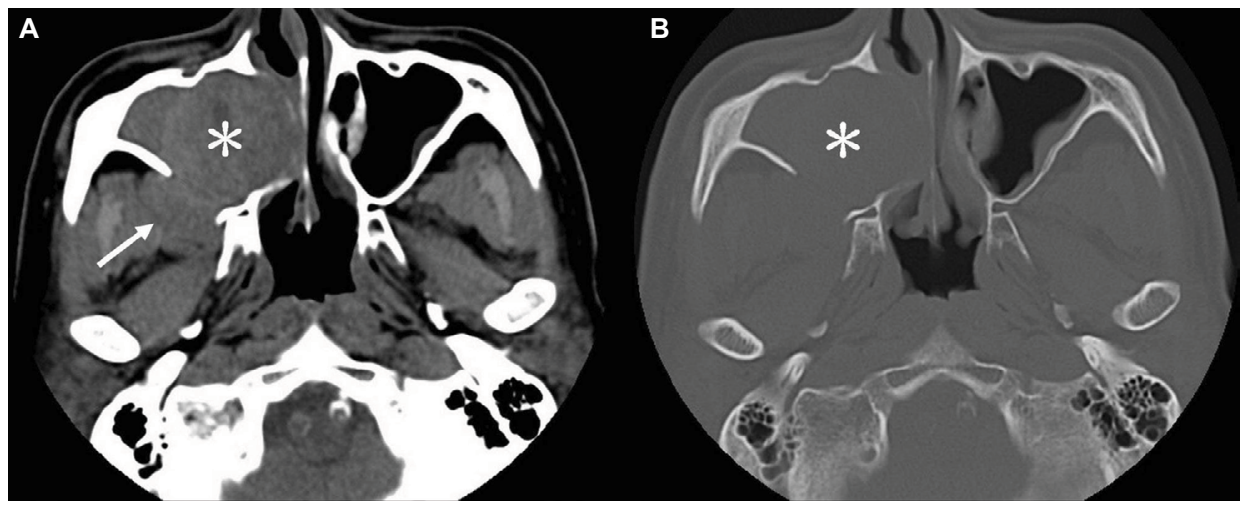

Figure 2 CT imaging of sinonasal angiomatous polyp.

Notes: Axial CT scan with a soft tissue algorithm (A) shows an expansile soft-tissue mass (asterisk) centered in the right maxillary sinus with interspersed hyperdense components. The mass extended into the right nasal cavity and the right infratemporal fossa (arrow). Axial CT with a bone algorithm (B) shows bony erosion of the medial and posterior maxillary sinus wall.

Abbreviation: $\mathrm{CT}$, computed tomography.

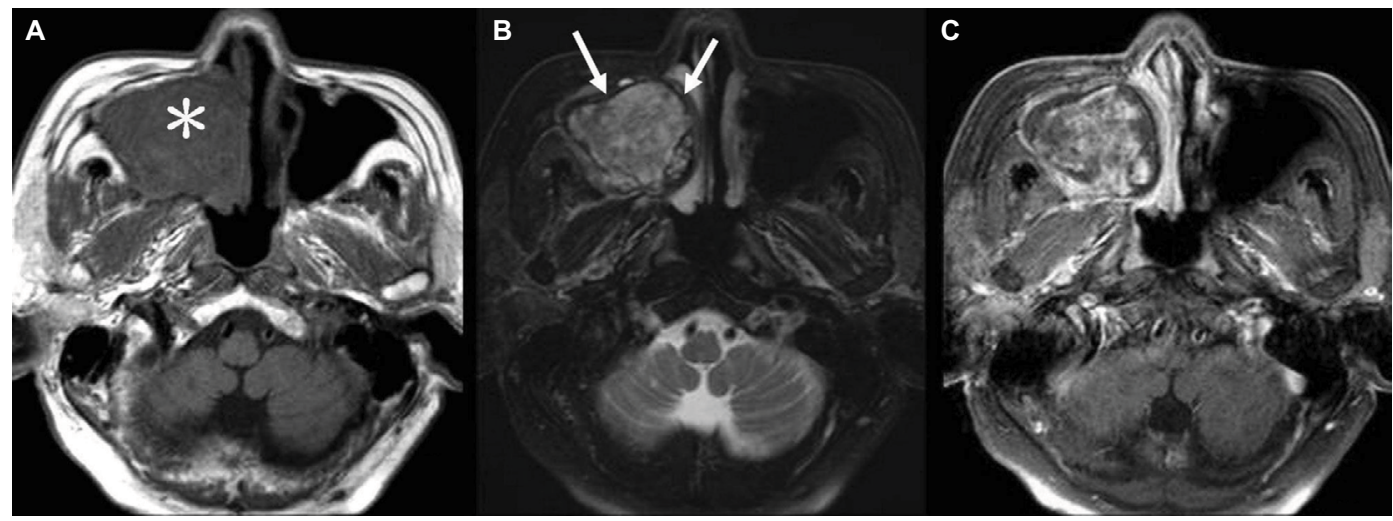

Figure 3 MR imaging of sinonasal angiomatous polyp.

Notes: Axial TI-weighted MR image (A) shows that a lobulated, heterogeneous mass (asterisk) with mildly hyperintense components was demonstrated in the right maxillary sinus. Axial fat-suppressed T2-weighted MR image (B) shows a well-defined mass with heterogenous central hyperintensity and peripheral hypointense rim (arrows). Axial fat-suppressed contrast-enhanced TI-weighted MR image $(\mathbf{C})$ shows heterogeneously avid contrast enhancement of the mass.

Abbreviation: MR, magnetic resonance. 


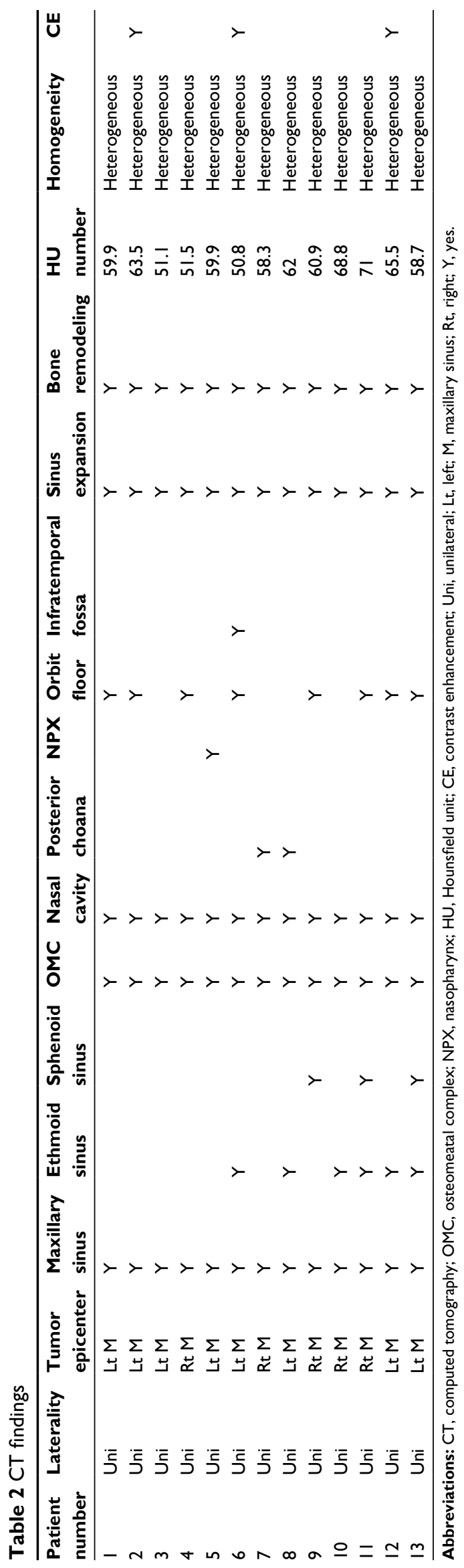

were observed on all the pulse sequences, especially T2WI (Figure 3). The imaging findings are summarized in Table 2.

\section{Pathological features}

On suspicion of sinonasal malignant tumor from the clinical and radiological findings, 12 patients underwent incisional biopsy of the unilateral sinonasal tumor before surgery. The pathology reports of these biopsy procedures noted blood clots, inflammatory polyps, and fibrous exudates. Postoperative final pathology findings were as follows. Grossly, the submitted tissues were fragmented soft to slightly elastic polypoid masses. On sectioning, the cut surfaces were glittering tan and yellow-brown in color, translucent with alternating zones of hemorrhage and edematous degeneration. Microscopically, all polyps from different patients shared remarkably similar histopathological features. The surfaces of the polyps were covered by pseudostratified respiratory-type epithelium with occasional focal squamous metaplasia. Beneath the surface epithelium was the hypocellular stroma expanded by edema and extravasated eosinophilic amorphous fibrin-like material. Seromucinous glands were seen at the base of or adjacent to the polyps but seldom in the proper polyp stroma. Instead, clusters of irregularly shaped and thin-walled blood vessels were seen. Areas of hemorrhage with hemorrhagic necrosis were also observed, and thrombus formation and neovascularization were found focally. The inflammatory infiltrate was generally mild but more pronounced in the areas of hemorrhagic necrosis and neovascularization (Figure 4).

\section{Discussion}

Sinonasal polyps are classified into several types: edematous, glandular, fibrous, cystic, and angiectatic or angiomatous. The nomenclature of SAPs is not consistent in the literature and includes granuloma telangiectaticum, vascular granuloma, organizing hematoma, cavernous hemangioma, hematoma-like mass of the antrum, pseudotumor, hemorrhagic necrotic polyp, and angioectatic or angiomatous polyp. ${ }^{5,8}$ The clinical, radiological, and pathological findings of the above-mentioned entities are all the same. In recent studies, the term "angiomatous polyp" has been suggested as being more suitable and compatible with the pathological features of extensive vascular proliferation, hemorrhage, and infarction. In our hospital, the diagnoses were also inconsistent and included inflammatory polyp with infarction and hemorrhagic or infarcted angiomatous polyp, ${ }^{6}$ and we enrolled the patients according to the detailed pathological description given earlier, which shared common clinical and radiological findings. 

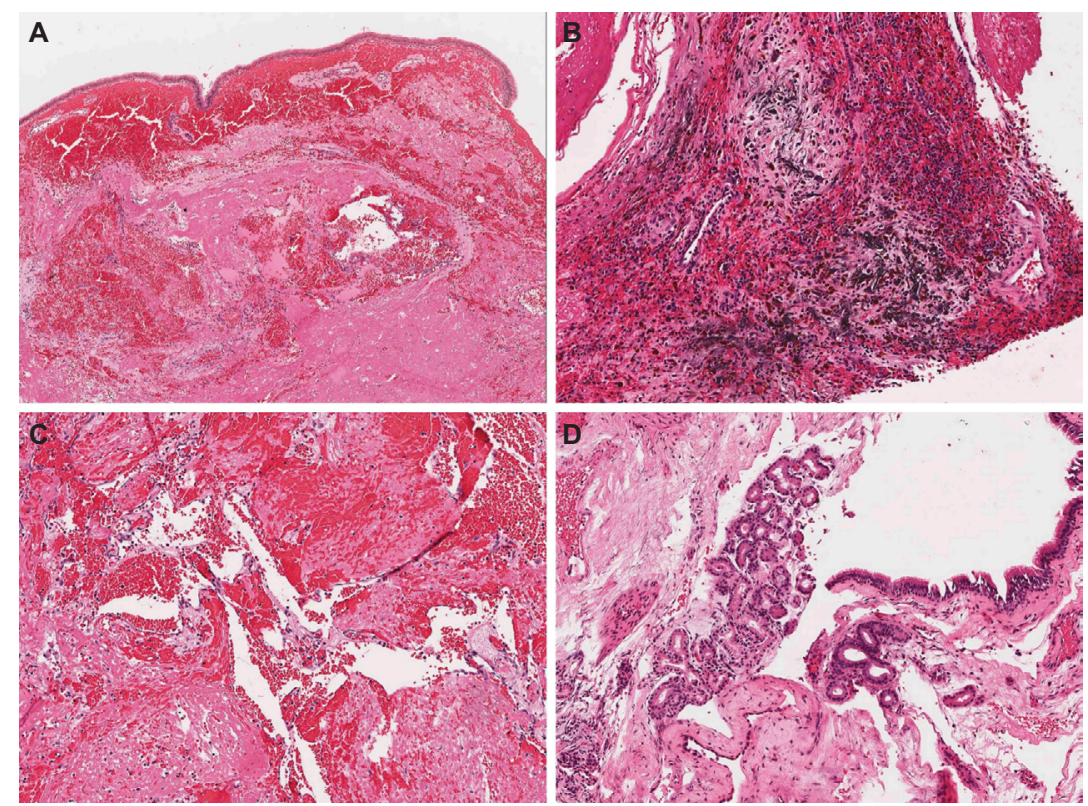

Figure 4 H\&E staining pathology pictures.

Notes: (A) Microphotograph showing a polyp covered by respiratory-type epithelium; the stroma shows marked hemorrhage and extravasation of eosinophilic fibrinoid material (40X). (B) Microphotograph shows an area with marked hemorrhage, neovascularization, and infiltration of chronic inflammatory cells (I00x). (C) Microphotograph shows clusters of irregularly shaped ectatic thin-walled vessels with hemorrhage and stromal fibrinoid material deposition (I00X). (D) Microphotograph shows seromucinous glands within adjacent respiratory mucosa, seen rarely in the proper polyp stroma (I00X).

Abbreviation: H\&E, hematoxylin and eosin.

Several hypotheses for the pathogenesis of SAPs have been reported in the literature. One is that SAP is derivative of an antrochoanal polyp. ${ }^{9}$ An antrochoanal polyp originates from the maxillary sinus and protrudes via the sinus ostium into the nasal cavity, which may extend posteriorly to the posterior choana and even the nasopharynx. Owing to the anatomical structure, a sinonasal polyp is more vulnerable to vascular compromise or strangulation at several sites, such as at the polyp pedicle, the sinus ostium, the posterior end of the inferior turbinate, the posterior choana, or the nasopharynx. ${ }^{2}$ Compression of the vessels results in vascular dilatation, stasis, edema, and ischemia of the polyp. This can also cause venous infarction, thrombosis formation, and subsequent neovascularization and fibrosis of the polyp, ${ }^{1,8}$ whereupon the term "angiomatous" was proposed. This process also accounts for the progressive expansion and regional bony destruction associated with SAP. ${ }^{5}$ We support this theory because all the SAPs in our patients originated from the maxillary sinus, involved the osteomeatal complex, and extended into the nasal cavity, with or without involvement of the posterior choana and nasopharynx, and all featured sinus expansion and bony destruction. Therefore, we suggest that the term SAP is more appropriate for the diagnosis and compatible with the pathogenesis. Notably, all our patients denied previous surgery or history of trauma, but six patients out of
13 had a history of diabetes mellitus and four were taking aspirin. Elevated blood sugar levels cause the blood vessels to be more susceptible to damage and formation of atherosclerosis. By blocking the production of prostaglandin, aspirin can dilate the vessels and prevent clotting, which may lead to hemorrhage. We believe that these conditions may account for the development of SAP in some of our patients.

The CT findings of SAP were not specific. The typical features are expansile mass in the sinus with bony wall destruction and remodeling. ${ }^{5}$ Some other benign lesions can exhibit similar bony erosion on CT imaging, such as juvenile angiofibroma, inverted papilloma, and hemangioma. ${ }^{8}$ Ding et al ${ }^{10}$ have reported that the vessel-like marked enhancement and progressive enhancement on two-phase helical CT scans are characteristic features of SAP, which could be a useful tool to make more confirmative diagnosis before surgery. We cannot always assume that bony destruction is a sign of malignancy. However, along with unilaterality, epistaxis, and nasal obstruction, clinicians and radiologists could easily mistake the lesions for malignancies. When encountered with patients with unilateral nasal symptoms, we rhinologists are always suspicious of neoplastic disease or foreign bodies. Hence, nearly half of our patients underwent MR studies for the differential diagnosis to rule out sinonasal malignancy. 
On MR imaging, slight hyperintensity on $\mathrm{T}^{1} \mathrm{WI}^{5}$ and a hypointense peripheral rim around the mass on T2WI, indicative of old hemorrhage, are more specific to the correct diagnosis of SAP. ${ }^{4}$

\section{Conclusion}

SAP is a rare variant of sinonasal polyp that mimics inverted papilloma, juvenile angiofibroma, and malignant tumor in its clinical and radiological aspects. The patients in our series most commonly presented with unilateral nasal obstruction, rhinorrhea, and epistaxis. The CT and MR images showed expansile sinonasal-occupying lesions with bony destruction and obstructive sinusitis in adjacent sinus cavities. The MR images provided more information to facilitate a correct diagnosis. Moreover, there were other clues to aid the differential diagnosis. When faced with patients who are older, taking aspirin, or who have diabetes mellitus, clinicians should remind themselves of the possibility of SAP. Most of all, an incisional biopsy to confirm the diagnosis before surgical treatment is helpful, whether preoperatively or intraoperatively. For patients with SAP, the treatment of choice is to completely remove the polypoid mass and restore the drainage system of all sinus cavities.

\section{Disclosure}

The authors report no conflicts of interest in this work.

\section{References}

1. De Vuysere S, Hermans R, Marchal G. Sinochoanal polyp and its variant, the angiomatous polyp: MRI findings. Eur Radiol. 2001;11(1): $55-58$.

2. Batsakis JG, Sneige N. Choanal and angiomatous polyps of the sinonasal tract. Ann Otol Rhinol Laryngol. 1992;101(7):623-625.

3. Sheahan P, Crotty PL, Hamilton S, Colreavy M, McShane D. Infarcted angiomatous nasal polyps. Eur Arch Otorhinolaryngol. 2005;262(3):225-230.

4. Wang YZ, Yang BT, Wang ZC, Song L, Xian JF. MR evaluation of sinonasal angiomatous polyp. AJNR Am J Neuroradiol. 2012;33(4):767-772.

5. Zou J, Man F, Deng K, Zheng Y, Hao D, Xu W. CT and MR imaging findings of sinonasal angiomatous polyps. Eur $J$ Radiol. 2014;83(3):545-551.

6. Huang CC, Lee TJ, Chang PH, Huang CC. Radiology quiz case 1. Infarcted angiomatous polyps of the maxillary sinus. Arch Otolaryngol Head Neck Surg. 2010;136(7):740, 742.

7. Yfantis HG, Drachenberg CB, Gray W, Papadimitriou JC. Angiectatic nasal polyps that clinically simulate a malignant process: report of 2 cases and review of the literature. Arch Pathol Lab Med. 2000;124(3):406-410.

8. Dai LB, Zhou SH, Ruan LX, Zheng ZJ. Correlation of computed tomography with pathological features in angiomatous nasal polyps. PLoS One. 2012;7(12):e53306.

9. Ceylan A, Asal K, Celenk F, Uslu S. An angiomatous nasal polyp: a very rare variant of sinochoanal nasal polyps. B-ENT. 2007;3(3):145-147.

10. Ding C, Wang Q, Guo Q, Wang Z, Lu X, Zhang J. Sinonasal angiomatous polyp: evaluation with 2-phase helical computed tomography. Medicine (Baltimore). 2015;94(29):e1196.
International Journal of General Medicine

\section{Publish your work in this journal}

The International Journal of General Medicine is an international, peer-reviewed open-access journal that focuses on general and internal medicine, pathogenesis, epidemiology, diagnosis, monitoring and treatment protocols. The journal is characterized by the rapid reporting of reviews, original research and clinical studies across all disease areas.

\section{Dovepress}

The manuscript management system is completely online and includes a very quick and fair peer-review system, which is all easy to use. Visit http://www.dovepress.com/testimonials.php to read real quotes from published authors. 